\title{
Comment on "An Investigation into the Performance of Particle Swarm Optimization with Various Chaotic Maps"
}

\author{
Yudong Zhang, ${ }^{1}$ Genlin Ji, ${ }^{1}$ Zhengchao Dong, ${ }^{2}$ Shuihua Wang, ${ }^{1}$ and Preetha Phillips ${ }^{3}$ \\ ${ }^{1}$ School of Computer Science and Technology, Nanjing Normal University, \\ Nanjing, Jiangsu 210023, China \\ ${ }^{2}$ Translational Imaging Division and MRI Unit, Columbia University and New York State Psychiatric Institute, \\ New York, NY 10032, USA \\ ${ }^{3}$ School of Natural Sciences and Mathematics, Shepherd University, Shepherdstown, WV 25443, USA
}

Correspondence should be addressed to Yudong Zhang; zhangyudong@njnu.edu.cn

Received 9 February 2015; Accepted 23 March 2015

Academic Editor: Marzio Pennisi

Copyright (c) 2015 Yudong Zhang et al. This is an open access article distributed under the Creative Commons Attribution License, which permits unrestricted use, distribution, and reproduction in any medium, provided the original work is properly cited.

This paper researched three definitions of Gauss map and found that the definition of "Gauss map" in the paper of Arasomwan and Adewumi may be incoherent with other publications. In addition, we analyzed the difference of continuous Gauss map and the floating-point Gauss map, and we pointed out that the floating-point simulation behaved significantly differently from the continuous Gauss map.

\section{Introduction}

Paper [1] is very welcome. The authors investigated the effect of nine chaotic maps on the performance of two variants of particle swarm optimization (PSO) algorithm as random inertia weight PSO (RIW-PSO) and linear decreasing inertia weight PSO (LDIW-PSO). Their simulation results showed that "the performances of those two variants were improved by many of the chaotic maps."

However, the authors give an inappropriate definition of Gauss map (Gaussian map) that some readers may get the mistaken understanding. There are currently three different types of Gauss maps for different disciplines. We will discuss them in what follows.

Definition 1. The first definition is used in differential geometry, where the "Gauss map" maps a surface in Euclidean space $R^{3}$ to the unit spheres $S^{2}$; that is, given a surface $X$ lying in $R^{3}$, the Gauss map is a continuous map $N: X \rightarrow S^{2}$ such that $N(p)$ is a unit vector orthogonal to $X$ at $p$, namely, the normal vector to $X$ at $p$.
Definition 2. The second definition of Gauss map is related to continued fractions and is used in programming, chaos, ergodic theory, and so forth. Its form is

$$
f(z)= \begin{cases}0, & z=0, \\ \frac{1}{z}-\left\lfloor\frac{1}{z}\right\rfloor, & \text { otherwise, }\end{cases}
$$

where the \lfloor\rfloor represents the floor function. Note that Corless [2] investigated this Gauss map and commented on it, "The Gauss map has been shown to be a good example of a chaotic discrete dynamical system"; however, "The numerical simulation of the map behaves significantly differently, in that the numerical simulation is not chaotic" (a more detailed discussion is offered in the Appendix).

Definition 3. The third definition of Gauss map is a chaotic series with the definition (Formula 3.42 in Anagnostopoulos [3] and Formula 3.3 in Saha and Das [4]) of

$$
x_{n+1}=\exp \left(-\alpha x_{n}^{2}\right)+\beta .
$$




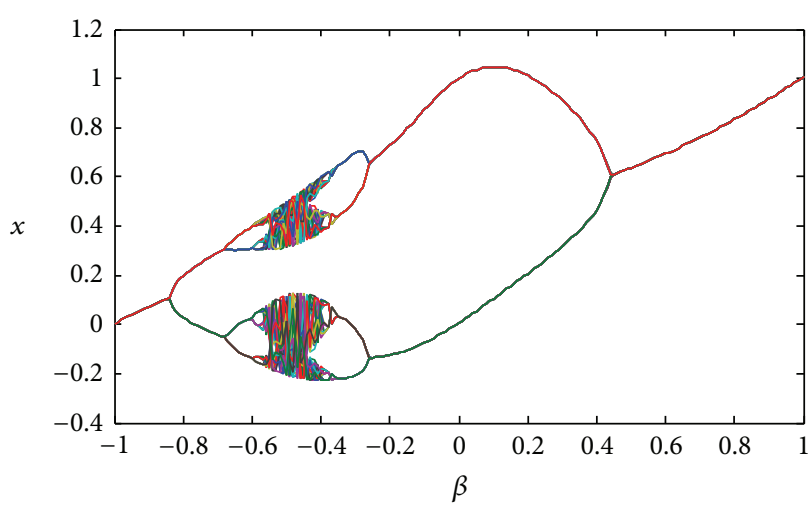

(a) $\alpha=4.9$

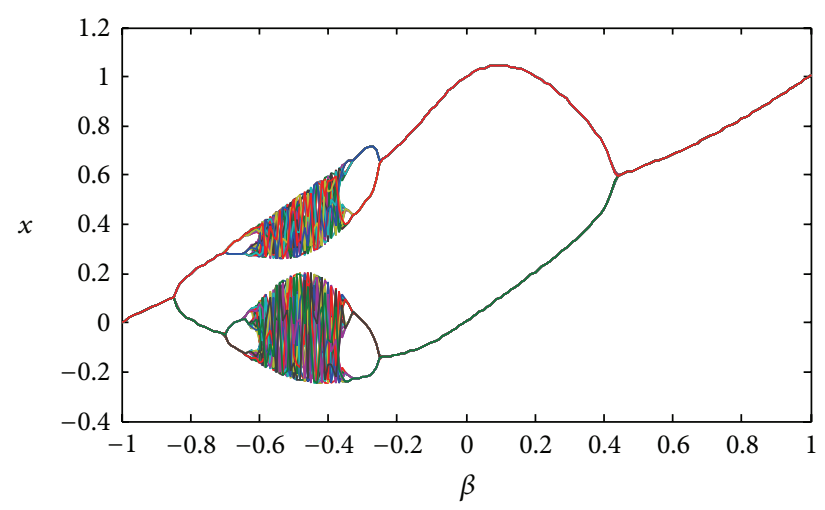

(b) $\alpha=5.2$

FIgURE 1: Bifurcation of Gauss/mouse map of Definition $2(x(0)=0.1)$.

This type of definition is also called "mouse map," since its bifurcation map resembles a mouse (see Figure 1).

Arasomwan's Definition. In Section 3.3, formula (9), page 3, Arasomwan and Adewumi [1] stated the following.

"3.3. Gaussian. This map is also known as Gauss or mouse map. It is defined as

$$
x_{k+1}= \begin{cases}0, & x_{k}=0, \\ \frac{1}{x_{k}} \bmod (1), & x_{k} \in(0,1),\end{cases}
$$

where $\left(1 / x_{k}\right) \bmod (1)=\left(1 / x_{k}\right)-\left\lfloor 1 / x_{k}\right\rfloor$ and $\lfloor z\rfloor$ denotes the largest integer less than $z$ which acts as a shift on the continued fraction representation of numbers."

\section{Discussion}

Arasomwan's definition of "Gauss map" [1] contains a paradox. From the context ("The map is also known as Gauss or mouse map"), we guess the third definition was appropriate, because this kind of Gauss map has a nickname of "Mouse map." From formula (9), we guess the second definition was appropriate, because formula (9) was similar to formula (1). It is suggested that the authors either remove the phrase of "Mouse map" or replace formula (9) with formula (2).

\section{Conclusion}

In spite of this issue, [1] is one of the best papers that we have ever read. We enjoyed reading it, which gave many thoughtprovoking and informative ideas especially in the results that "intermittency map" performed best among all chaotic maps.

\section{Appendix}

Suppose Arasomwan and Adewumi [1] used Definition 2 (in what follows, the Gauss map denotes Definition 2); another paradox was raised between the continuous model (see formula (1)) and the floating-point simulation. Unlike common sense, formidable difficulties and considerable changes happen in simulating the Gauss map. To support it, let us revisit the two important properties of the continuous Gauss map [5].

(C1) The orbit of $\left\{x_{k}\right\}$ of every rational initial point $x_{0}$ goes to zero in a finite number of iterations.

(C2) The Lyapunov exponent of the map is, for almost all initial points, $\pi^{2} /(6 \log 2)=2.3731 \ldots$ but is undefined for rational initial points and is different for each quadratic irrational initial point.

The first property is proved by "Gauss's continuous fraction." In the second property, the Lyapunov exponent characterizes the rate of separation of infinitesimally close trajectories in a dynamical system, and a positive maximal Lyapunov exponent indicates the system is chaotic. For the floating-point simulation, we found the two properties of continuous model were changed by the following facts.

(S1) On a HP28S calculator, starting from $x_{0}=0.73$ (it is a rational number) and using $10^{5}$ iterations of the floating-point Gauss map, we get a computed Lyapunov exponent equal to 2.36992 [5].

(S2) Irrational points are impossible to be represented in a floating-point machine, remembering the concept of smallest machine representable number.

From the conflicts between $(\mathrm{Cl})$ and $(\mathrm{S} 1)$ and between (C2) and (S2), we guess the reason is that the floating-point arithmetic destroys the inherit chaos while the round-off error introduces the spurious chaos, when transforming the continuous Gauss map to a floating-point simulation.

Let us revisit [1], in which they used floating-point simulation in the experiment while giving a continuous mathematical model of (9) in the methodology. It is suggested that the authors should give a detailed description of the transform from continuous Gauss map model to floatingpoint Gauss map model in their future work. 


\section{Conflict of Interests}

The authors have no conflict of interests to disclose with regard to the subject matter of this paper.

\section{Acknowledgments}

The authors give their thanks to the editors for giving them an opportunity to share their ideas with the readers. This paper was supported by NSFC (no. 610011024), Program of Natural Science Research of Jiangsu Higher Education Institutions of China (no. 14KJB520021), Jiangsu Key Laboratory of 3D Printing Equipment and Manufacturing (BM2013006), and Nanjing Normal University Research Foundation for Talented Scholars (no. 2013119XGQ0061).

\section{References}

[1] A. M. Arasomwan and A. O. Adewumi, "An investigation into the performance of particle swarm optimization with various chaotic maps," Mathematical Problems in Engineering, vol. 2014, Article ID 178959, 17 pages, 2014.

[2] R. M. Corless, "Continued fractions and chaos," The American Mathematical Monthly, vol. 99, no. 3, pp. 203-215, 1992.

[3] K. N. Anagnostopoulos, Computational Physics: A Practical Introduction to Computational Physics and Scientific Computing, 2014.

[4] L. M. Saha and M. K. Das, "Bifurcation and chaos measure in some discrete dynamical systems," Journal of Mathematical and Computational Science, vol. 3, no. 1, pp. 150-166, 2013.

[5] R. M. Corless, "What good are numerical simulations of chaotic dynamical systems?" Computers \& Mathematics with Applications, vol. 28, no. 10-12, pp. 107-121, 1994. 


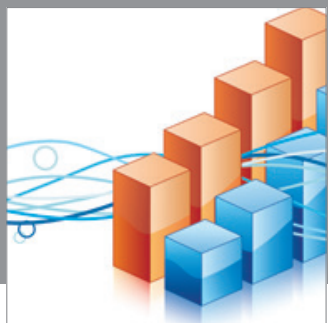

Advances in

Operations Research

mansans

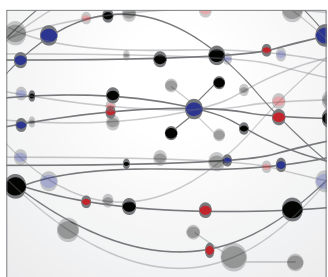

The Scientific World Journal
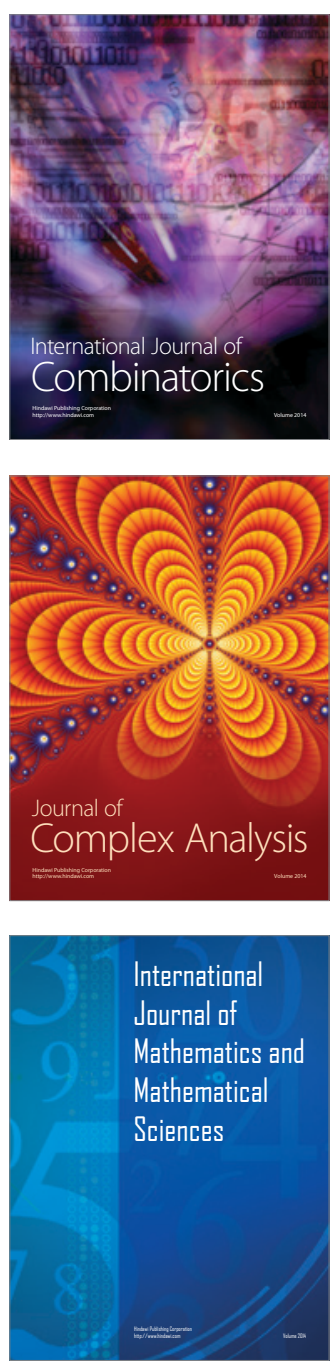
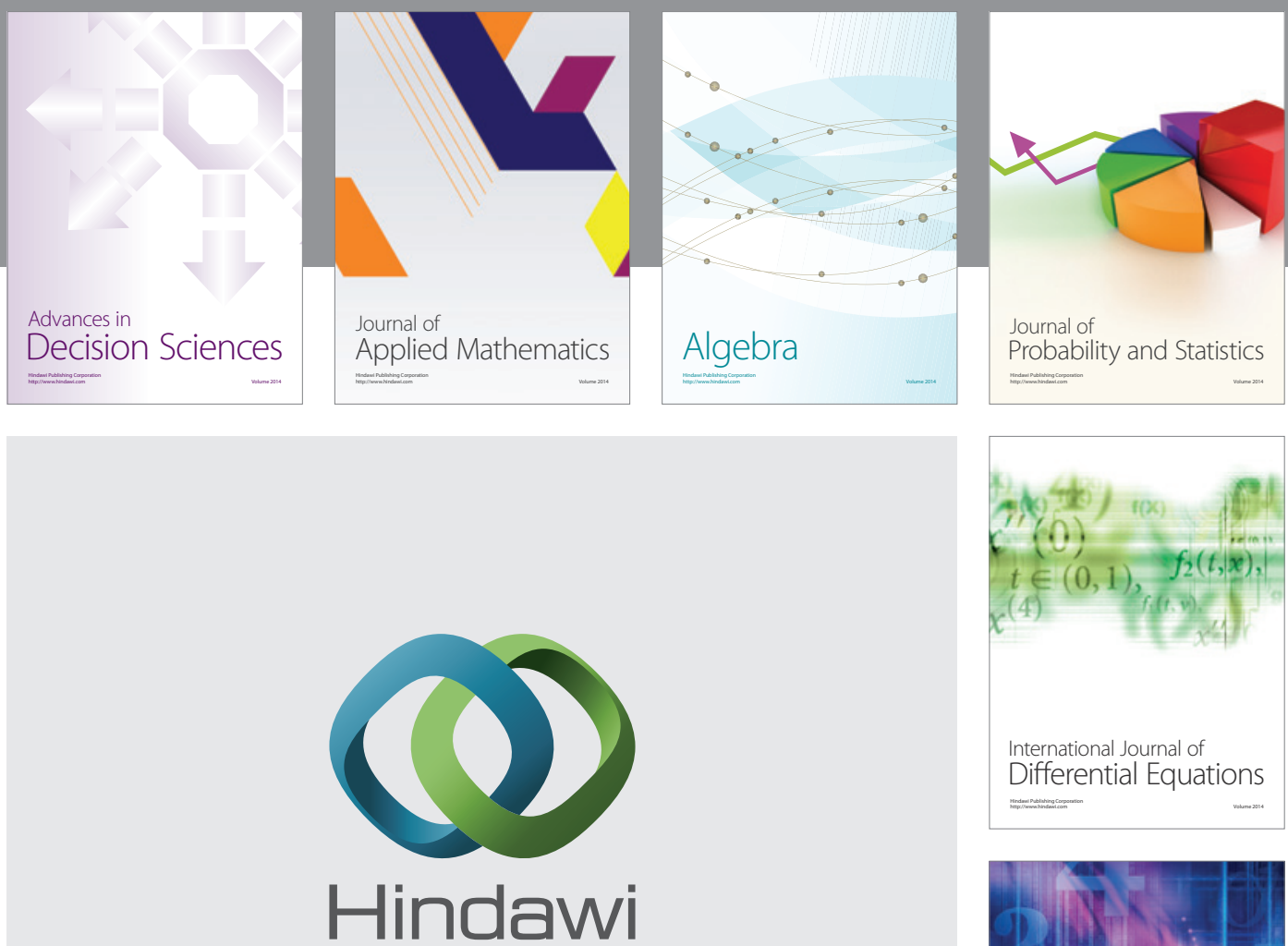

Submit your manuscripts at http://www.hindawi.com
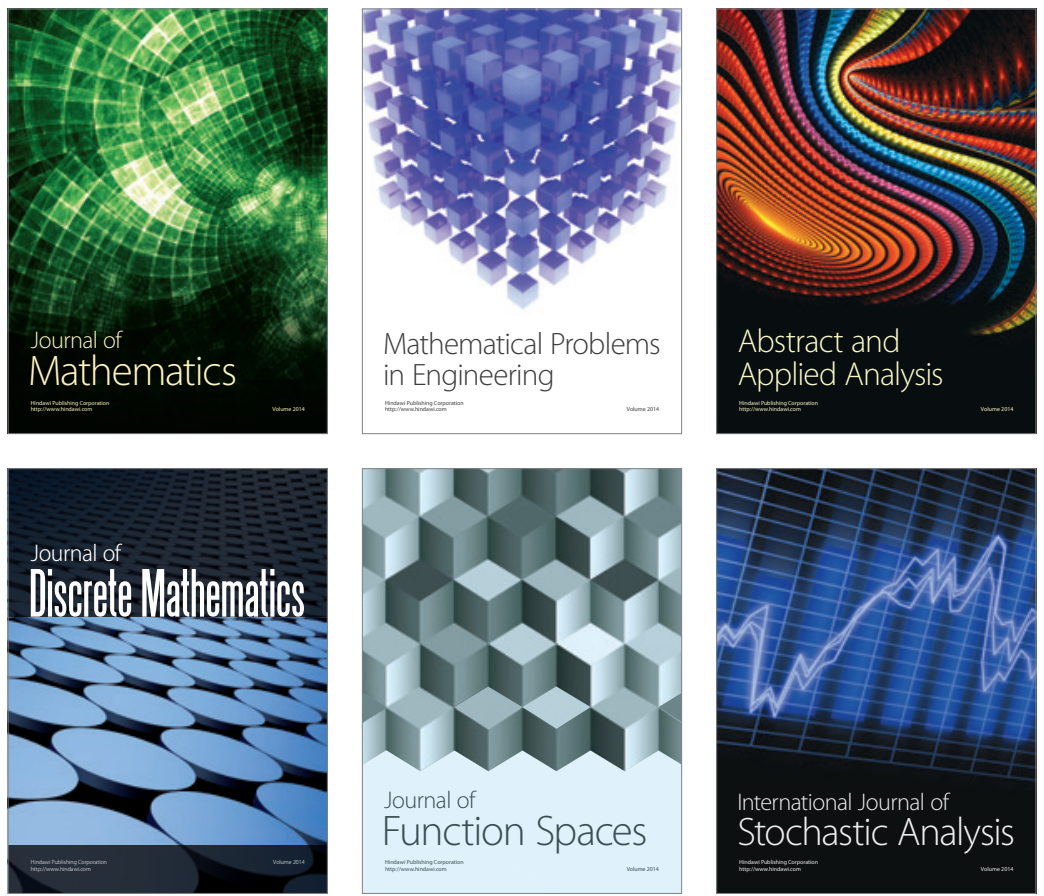

Journal of

Function Spaces

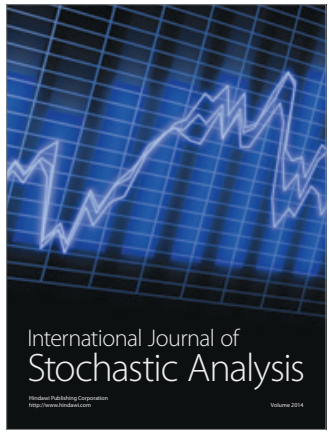

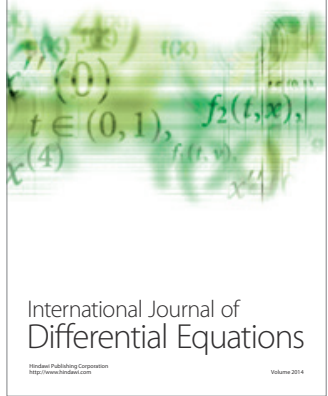
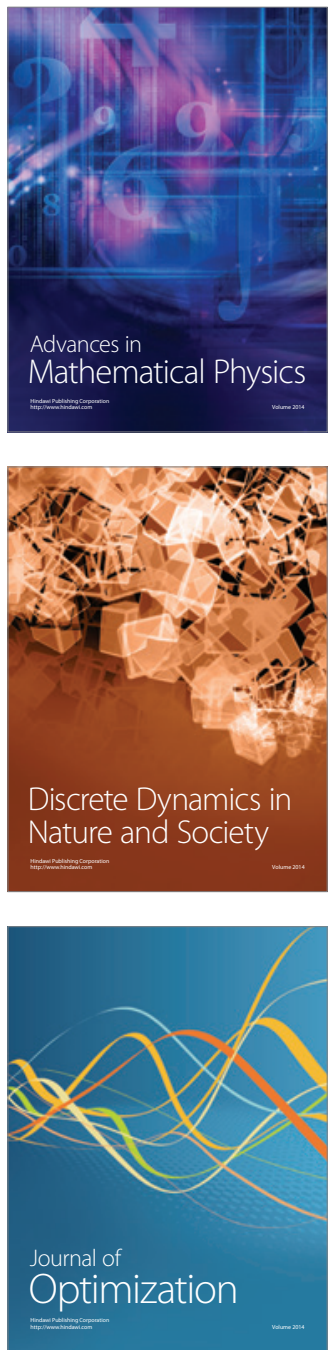